\title{
EXISTE RELAÇÃO DA VIA DE PARTO COM A AMAMENTAÇÃO NA PRIMEIRA HORA DE VIDA?
}

\author{
Is there a relation between mode of delivery and breastfeeding in the first hour of life?
}

\section{¿Hay relación entre la vía de parto y el amamantamiento en la primera hora de vida?}

\section{Guilherme Tavares de Arruda}

Universidade Federal de Santa Maria - UFSM - Santa Maria (RS) - Brasil

\section{Sabrina Cabreira Barreto}

Universidade Federal de Santa Maria - UFSM - Santa Maria (RS) - Brasil

Vanessa Lago Morin

Universidade Federal de Santa Maria - UFSM - Santa Maria (RS) - Brasil

\section{Gustavo do Nascimento Petter}

Universidade Franciscana - UFN - Santa Maria (RS) - Brasil

\section{Melissa Medeiros Braz}

Universidade Federal de Santa Maria - UFSM - Santa Maria (RS) - Brasil

\section{Hedioneia Maria Foletto Pivetta}

Universidade Federal de Santa Maria - UFSM - Santa Maria (RS) - Brasil

\section{RESUMO}

Objetivo: Investigar a relação da via de parto com fatores sociodemográficos e amamentação. Métodos: Pesquisa quantitativa, transversal e descritiva, realizada com mães de crianças de 0 a 2 anos que acessaram unidades de saúde em uma campanha de vacinação em uma cidade no Sul do Brasil, em junho de 2013. Participaram 905 mulheres, divididas em dois grupos, conforme via de parto: grupo parto vaginal ( $\mathrm{n}=334$ ) e grupo cirurgia cesariana ( $\mathrm{n}=571$ ). Responderam a um questionário contendo informações sociodemográficas (idade, cor, escolaridade, situação conjugal, classe social e área de residência) e relacionadas à amamentação, como tempo de aleitamento materno exclusivo, amamentação na primeira hora de vida e sucesso da amamentação. Utilizou-se Teste Z para amostras independentes de variáveis paramétricas e Teste $\mathrm{Z}$ de Kolmogorov-Smirnov quando não-paramétricas, com nível de significância $\mathrm{p}=0,05$. Resultados: $\mathrm{O}$ grupo parto vaginal era de cor branca $(\mathrm{n}=210 ; 62,87 \%)$, com união estável $(\mathrm{n}=124 ; 37,13 \%)$, ensino fundamental $(\mathrm{n}=177 ; 52,99 \%)$, da classe social $\mathrm{C} 1(\mathrm{n}=114 ; 34,13 \%)$ e residente na área urbana $(n=321 ; 96,11 \%)$. O grupo cirurgia cesariana constituiu-se de mulheres brancas $(n=439 ; 76,88 \%)$, casadas $(n=316 ; 55,34 \%)$, com ensino médio $(\mathrm{n}=223 ; 39,05 \%)$, da classe social B2 $(\mathrm{n}=184 ; 32,22 \%)$ e residentes na área urbana $(\mathrm{n}=544 ; 95,27 \%)$. O aleitamento materno na primeira hora de vida predominou no grupo parto vaginal $(\mathrm{n}=265 ; 79,34 \%)$, com diferença significativa $(\mathrm{p} \leq 0,001)$. Conclusão: A via de parto possuiu relação com amamentação na primeira hora de vida no grupo estudado, bem como com a idade, a cor da pele, a escolaridade e a classe social maternas.

Descritores: Parto; Aleitamento materno; Dados Demográficos.

\section{ABSTRACT}

Objective: To investigate the relation between mode of delivery, sociodemographic factors and breastfeeding. Methods: A quantitative, crosssectional and descriptive study, carried out with mothers of children aged 0 to 2 years seen at health units during a vaccination campaign in a city in the South of Brazil in June 2013. Participants were 905 women, divided into two groups, according to the delivery method: vaginal delivery group $(n=334)$ and Cesarean section group $(n=571)$. They answered a questionnaire comprising sociodemographic information (age, color, schooling, marital status, social class and area of residence) and breastfeeding-related data, such as duration of exclusive breastfeeding, breastfeeding in the first hour of life, and breastfeeding success. Z-Test was applied for independent samples of parametric variables and Kolmogorov-Smirnov Z-Test was used for non-parametric variables, with significance level at $p=0.05$. Results: The vaginal delivery group was white $(n=210,62.87 \%)$, with a stable union $(n=124,37.13 \%)$, elementary school $(n=177,52.99 \%)$, social grade C1 $(n=114 ; 34.13 \%)$ and resident in the urban area $(n=321 ; 96.11 \%)$. C-section group consisted of white women $(n=439,76.88 \%)$, married $(n=316,55.34 \%)$, high school graduates $(n=223,39.05 \%)$, social grade $B 2(n=184 ; 32.22 \%)$ and residents in the urban area $(n=544,95.27 \%)$. Breastfeeding in the first hour of life predominated in the vaginal delivery group $(n=265 ; 79.34 \%)$, with a significant difference ( $p \leq 0.001)$. Conclusion: 
In the studied group, the mode of delivery was associated to breastfeeding in the first hour of life, and to maternal age, skin color, schooling and social class as well.

Descriptors: Childbirth; Breastfeeding; Demographic Data.

\section{RESUMEN}

Objetivo: Investigar la relación entre la vía de parto con los factores sociodemográficos y el amamantamiento. Métodos: Investigación cuantitativa, transversal y descriptiva realizada con madres de niños entre 0 y 2 años que acudieron a unidades básicas de salud en una campaña de vacunación de una ciudad del Sur de Brasil en junio de 2013. Participaron 905 mujeres divididas en dos grupos según la vía del parto: grupo parto vaginal $(n=334)$ y grupo cirugía cesárea $(n=571)$. Las mujeres contestaron un cuestionario con informaciones sociodemográficas (edad, color, escolaridad, situación conyugal, clase social y zona de vivienda) y las relacionadas con el amamantamiento como el tiempo de amamantamiento materno exclusivo, amamantamiento en la primera hora de vida y el éxito del amamantamiento. Se utilizó el test $Z$ para muestras independientes de las variables paramétricas y el test $Z$ de Kolmogorov-Smirnov para las no paramétricas con nivel de significación de $p=0,05$. Resultados: El grupo parto vaginal era de color blanco ( $n=210 ; 62,87 \%)$, parejas de hecho ( $n=124$; $37,13 \%)$, con educación primaria $(n=177 ; 52,99 \%)$, de la clase social C1 $(n=114 ; 34,13 \%)$ y residente del área urbana $(n=321 ; 96,11 \%)$. El grupo cirugía cesárea se ha constituido de mujeres blancas ( $n=439 ; 76,88 \%)$, casadas $(n=316 ; 55,34 \%)$, con educación secundaria ( $n=223$; $39,05 \%)$, de la clase social B2 ( $n=184 ; 32,22 \%)$ y residentes del área urbana $(n=544 ; 95,27 \%)$. El amamantamiento materno en la primera hora de vida ha predominado en el grupo parto vaginal ( $n=265 ; 79,34 \%)$ con diferencia significativa ( $\leq \leq 0,001)$. Conclusión: La vía del parto tiene relación con el amamantamiento en la primera hora de vida en el grupo investigado así como con la edad, el color de la piel, la escolaridad y la clase social maternas.

Descriptores: Parto; Lactancia Materna; Dados Demográficos.

\section{INTRODUÇÃO}

A via de parto é uma decisão a ser tomada em conjunto pela mulher e pelo médico, sendo baseada na preferência, mas principalmente nas necessidades da parturiente. Nesse sentido, muitas vezes, a escolha pelo parto vaginal deve-se por possuir menor risco de intercorrências e ser de recuperação rápida. Em contrapartida, a opção pela cesárea ocorre por ser mais rápida, indolor e seguir recomendação médica ${ }^{(1)}$.

No Brasil, em 2015, as taxas de cirurgias cesarianas caíram 1,5 pontos percentuais no país. É a primeira vez que este procedimento decresce desde $2010^{(2)}$. Antes de 2015, o Brasil apresentava aumento significativo do número de cesarianas, chegando a mais de $50 \%$ do total de nascimentos, o que tornava o país líder mundial neste tipo de cirurgia ${ }^{(3)}$.

Os altos índices nacionais de cesáreas podem ser explicados, principalmente, pela centralização individual dos profissionais na escolha da via de parto, pelas características sociodemográficas e culturais da mulher, e pelas características da assistência pré-natal $^{(4)}$.

Sabe-se que, por ser um procedimento cirúrgico, a cesariana pode causar complicações maternas graves, sendo indicada somente quando necessário. Além disso, essa via de parto também gera consequências ao recém-nascido, aumentando a chances de prematuridade iatrogênica e internação em centros de terapia intensiva neonatal ${ }^{(5)}$. Há ainda estudos que sugerem que a operação cesariana esteja associada à interferência na amamentação na primeira hora de vida ${ }^{(6,7)}$.

Os primeiros 60 minutos de vida do bebê após o nascimento, chamado de golden hour, representam o período no qual são realizadas intervenções para minimizar as complicações neonatais ${ }^{(8)}$. Dentre tais intervenções, o contato pele a pele e a amamentação na primeira hora de vida promovem vínculo entre mãe e bebê e estimulam o reflexo de sucção da criança ${ }^{(9)}$. Dessa forma, medidas de promoção do aleitamento materno (AM) e do contato pele a pele na primeira hora de vida são necessárias para a maior taxa de sobrevida do neonato e menores índices de desmame precoce ${ }^{(10)}$.

Em contraponto, a cirurgia cesariana é referida como fator negativo sobre a amamentação, sendo relacionada ao desmame precoce e ao menor tempo de aleitamento materno exclusivo (AME) ${ }^{(11)}$.

Considerando-se o papel da equipe obstétrica na promoção da saúde do binômio mãe-bebê, é de fundamental importância conhecer a relação entre via de parto e amamentação, visando implementar estratégias adequadas para cada via de parto, a fim de garantir o sucesso do AM. Já entre fatores sociodemográficos e amamentação, a relação de tais características com a via de parto é pouco explorada. Dessa forma, o objetivo deste estudo foi investigar a relação da via de parto com fatores sociodemográficos e a amamentação.

\section{MÉTODOS}

Trata-se de uma pesquisa quantitativa, transversal, com abordagem exploratória e descritiva, desenvolvida junto a Unidades 
Básicas de Saúde (UBS) e Unidades de Estratégia de Saúde da Família (ESF), totalizando 24 serviços de saúde da Atenção Básica (do total de 32) de uma cidade no Sul do Brasil.

A coleta de dados ocorreu nos dias de campanha de vacinação contra a poliomielite, em 2013, executada pela Vigilância em Saúde do Município em parceria com o Ministério da Saúde. Incluíram-se no estudo mulheres, mães de crianças de 0 a 2 anos de idade, residentes na cidade onde a pesquisa foi realizada, que acessaram as Unidades de Saúde e unidades móveis do município nos dias de campanha.

Um questionário adaptado ${ }^{(12)}$ padronizado e pré-codificado foi aplicado pelo pesquisador às mães após a vacinação da criança.

Para este estudo, exploraram-se as variáveis do questionário relacionadas à via de parto; aos fatores sociodemográficos da mãe, como idade, cor da pele, escolaridade, situação conjugal, classe social ${ }^{(13)}$ e área de residência; e os fatores de amamentação, como amamentação na primeira hora de vida, sucesso na amamentação e tempo de AME (em meses).

Considerou-se a via de parto (vaginal ou cesáreo) como a variável-desfecho, sendo julgado como sucesso na amamentação o AME por, no mínimo, seis meses, como recomendado pela $\mathrm{OMS}^{(14)}$.

Para o cálculo do tamanho da amostra para desfecho AME, foram utilizados os seguintes parâmetros: intervalo de confiança (IC) $95 \%$, poder de $80 \%$, prevalência de AME de $34 \%$, conforme pesquisa realizada pelo município, por ocasião da II Pesquisa de Prevalência de Aleitamento Materno nas Capitais Brasileiras e Distrito Federal ${ }^{(12)}$ e RR1:2, obtendo-se uma amostra de 1.015 mães.

Após revisão e codificação dos dados, eles foram duplamente digitados e, posteriormente, comparados. A análise dos dados envolveu procedimentos de estatística descritiva e análises bivariadas para controle de eventuais fatores de confusão. Para análise da normalidade dos dados, utilizou-se o teste de Kolmogorov-Smirnov. Em seguida, empregou-se o teste qui-quadrado para comparações entre grupos em relação às variáveis categóricas. Para comparação entre médias, o Test $Z$ avaliou amostras independentes quando as variáveis eram paramétricas e o Teste $\mathrm{Z}$ de Kolmogorov-Smirnov quando eram não-paramétricas. Adotou-se o nível de significância de 0,05 para todos os testes e as análises ocorreram por meio do software SPSS, versão 15.0.

As avaliações somente foram efetivadas após o aceite e assinatura do Termo de Consentimento Livre e Esclarecido (TCLE), em atendimento à Resolução n ${ }^{\circ}$ 466/2012 do Conselho Nacional de Saúde. Esta pesquisa recebeu aprovação do Comitê de Ética em Pesquisa com Seres Humanos da Universidade Federal de Santa Maria, sob Parecer nº 129.893.

\section{RESULTADOS}

Das 1.015 mães avaliadas, 110 foram excluídas por não responderem ao instrumento de pesquisa em sua totalidade, restando 905 participantes. Estas foram divididas entre os grupos, conforme via de parto: grupo parto vaginal (GV) e grupo cirurgia cesariana (GC). O número de participantes no GV foi 334 (36,91\%) e no GC, 571 (63,09\%).

Os dados sociodemográficos das participantes estão apresentados na Tabela I, salientando que a média de idade do GC foi maior que a do GV.

A maiorias das mulheres do GV era de cor branca ( $\mathrm{n}=210 ; 62,87 \%)$, em união estável ( $\mathrm{n}=124 ; 37,13 \%)$, com escolaridade no nível de ensino fundamental $(\mathrm{n}=177 ; 52,99 \%)$, pertencentes à classe social $\mathrm{C} 1(\mathrm{n}=114 ; 34,13 \%)$ e residentes na área urbana $(\mathrm{n}=321 ; 96,11 \%)$. Já o GC constituiu-se de mulheres majoritariamente brancas $(\mathrm{n}=439 ; 76,88 \%)$, casadas $(\mathrm{n}=316 ; 55,34 \%)$, com escolaridade no nível de ensino médio $(\mathrm{n}=223 ; 39,05 \%)$, de classe social B2 $(\mathrm{n}=184 ; 32,22 \%)$ e residentes na área urbana $(\mathrm{n}=544 ; 95,27 \%)$ (Tabela I).

A análise dos dados demonstrou diferença entre os grupos com a idade materna, cor da pele, escolaridade e classe social da mãe $(\mathrm{p} \leq 0,001)$. Contudo, a situação conjugal e a área em que vive não pareceram estar relacionadas à via de parto.

$\mathrm{Na}$ análise da relação da amamentação com a via de parto, encontrou-se diferença significativa somente para a amamentação na primeira hora de vida $(\mathrm{p} \leq 0,001)$, como apresentado na Tabela II. Isto refletiu em maior número de bebês sendo amamentados na primeira hora entre as mães do GV comparadas às mães do GC. Já no que diz respeito às variáveis "sucesso na amamentação" (considerando sucesso na amamentação a AME durante seis primeiro meses de vida do bebê) e "tempo de AME", não foi encontrada associação com a via de parto e, em ambos os grupos, encontraram-se baixos índices de sucesso na amamentação e baixa média do tempo de AME.

\section{DISCUSSÃO}

O estudo em questão apresentou uma alta taxa de cirurgia cesariana (63,09\%) em relação ao parto vaginal. Dados semelhantes foram encontrados em outro estudo realizado no Sul, em que se verificou que mais de $60 \%$ dos partos realizados em Santa Catarina, no ano de 2012, foram via cirurgia cesariana ${ }^{(15)}$.

Diferentemente de outros países, como os Estados Unidos e a Itália, onde as taxas de cirurgia cesariana permanecem em torno de $30 \%{ }^{(16)}$, o Brasil possui índices superiores a $50 \%^{(3)}$. As altas taxas de cirurgia cesariana são mais notáveis em países menos desenvolvidos, como o Brasil, onde fatores culturais, sociodemográficos e econômicos interferem na escolha da via de 
parto $^{(16)}$. Dessa forma, é importante ressaltar as altas taxas de morbimortalidade materna e neonatal que as cesáreas acarretam ao sistema de saúde ${ }^{(17,18)}$.

Tabela I - Caracterização sociodemográfica das mães entrevistadas em relação à via de parto. Santa Maria, Rio Grande do Sul, Brasil, 2013.

\begin{tabular}{|c|c|c|c|}
\hline Caracterização sociodemográfica & GV $(n=334)$ & GC $(n=571)$ & $\mathbf{p}^{*}$ \\
\hline Idade (anos) Média (DP) & $26,51 \pm 7,45$ & $29,27 \pm 6,55$ & $0,001^{* *}$ \\
\hline $\begin{array}{l}\text { Cor } \\
\text { Branca } \\
\text { Parda } \\
\text { Preta } \\
\text { Amarela } \\
\text { Indígena }\end{array}$ & $\begin{array}{c}\mathbf{n}(\%) \\
210(62,87) \\
69(20,66) \\
47(14,07) \\
3(0,90) \\
2(0,60)\end{array}$ & $\begin{array}{c}\mathbf{n}(\%) \\
439(76,88) \\
68(11,91) \\
44(7,71) \\
6(1,05) \\
4(0,70)\end{array}$ & $0,001^{* *}$ \\
\hline $\begin{array}{l}\text { Escolaridade } \\
\text { Sem escolaridade } \\
\text { Ensino fundamental } \\
\text { Ensino médio } \\
\text { Ensino superior incompleto } \\
\text { Ensino superior completo }\end{array}$ & $\begin{array}{c}\mathbf{n}(\%) \\
2(0,60) \\
177(52,99) \\
118(35,33) \\
14(4,19) \\
17(5,09)\end{array}$ & $\begin{array}{c}\mathbf{n}(\%) \\
2(0,35) \\
135(23,64) \\
223(39,05) \\
64(11,21) \\
142(24,87)\end{array}$ & $0,001^{* *}$ \\
\hline $\begin{array}{l}\text { Situação conjugal } \\
\text { União estável } \\
\text { Casada } \\
\text { Solteira } \\
\text { Separada } \\
\text { Viúva }\end{array}$ & $\begin{array}{c}\mathbf{n}(\mathbf{\%}) \\
124(37,13) \\
113(33,83) \\
85(25,45) \\
10(2,99) \\
2(0,60)\end{array}$ & $\begin{array}{c}\mathbf{n}(\mathbf{\%}) \\
154(26,97) \\
316(55,34) \\
85(14,89) \\
13(2,28) \\
2(0,35)\end{array}$ & 0,600 \\
\hline $\begin{array}{c}\text { Classe social } \\
\text { Classe A1 } \\
\text { Classe A2 } \\
\text { Classe B1 } \\
\text { Classe B2 } \\
\text { Classe C1 } \\
\text { Classe C2 } \\
\text { Classe D } \\
\text { Classe E }\end{array}$ & $\begin{array}{c}\mathbf{n}(\mathbf{\%}) \\
0(0) \\
2(0,60) \\
14(4,19) \\
79(23,65) \\
114(31,43) \\
94(28,14) \\
26(7,78) \\
4(1,20)\end{array}$ & $\begin{array}{c}\mathbf{n}(\mathbf{\%}) \\
4(0,70) \\
45(7,88) \\
95(16,64) \\
184(32,22) \\
129(22,59) \\
79(13,84) \\
29(5,08) \\
3(0,53)\end{array}$ & $0,001^{* *}$ \\
\hline $\begin{array}{l}\text { Área de residência } \\
\text { Urbana } \\
\text { Rural }\end{array}$ & $\begin{array}{c}\mathbf{n}(\mathbf{\%}) \\
321(96,11) \\
12(3,59)\end{array}$ & $\begin{array}{c}\mathbf{n}(\mathbf{\%}) \\
544(95,27) \\
25(4,38)\end{array}$ & 0,564 \\
\hline
\end{tabular}

Variáveis apresentadas em Média \pm Desvio padrão (DP), ou n= número absoluto e \% = frequência. GV: Grupo parto vaginal; GC: Grupo parto cesáreo. "Teste Z. ${ }^{* *} p \leq 0,05$.

Tabela II - Análise dos fatores de amamentação em relação à via de parto. Santa Maria, Rio Grande do Sul, Brasil, 2013.

\begin{tabular}{lccc}
\hline Fatores de amamentação & GV & GC & $\boldsymbol{p}^{*}$ \\
\hline Amamentação na primeira hora de vida & $265(79,34)$ & $397(69,53)$ & $0,001^{*}$ \\
Sucesso da amamentação** $^{* * * *}$ & $86(25,75)$ & $158(27,57)$ & 0,438 \\
Tempo de AME (meses) $^{* * *}$ & $3,14 \pm 3,19$ & $3,22 \pm 3,27$ & 0,889 \\
\hline
\end{tabular}

Variáveis apresentadas em Média \pm DP, ou n (\%). GV: Grupo parto vaginal. GC: Grupo parto cesárea. AME: aleitamento materno exclusivo. ${ }^{*} p \leq 0,05,{ }^{* *}$ Considera-se sucesso na amamentação a AME durante seis meses de vida do bebê, ${ }^{* * *}$ Teste Z.

No presente estudo, algumas variáveis parecem influenciar a tomada de decisão pela via de parto, assim como em um estudo realizado sobre a relação da via de parto com variáveis temporais e sociodemográficas em mulheres brasileiras, em que se observou que a taxa de cesariana é menor em mães mais jovens e de baixa escolaridade, mas aumenta conforme idade e anos de estudo ${ }^{(17)}$. Esses resultados são semelhantes aos encontrados na presente pesquisa, visto a média de idade ser de 26,51 anos entre as mulheres do GV. Embora a média de idade das mães do atual estudo não seja tão jovem, a diferença do GV e do GC foi significativa para o tipo de parto, sendo, portanto, aqui considerado como "mães jovens". 
Quanto à escolaridade, observou-se, na atual investigação, que quanto menor seu nível, maior a probabilidade da ocorrência de parto por via vaginal. No GV, mais de 50\% das mães estudaram apenas até o ensino fundamental, enquanto no GC houve predomínio de mulheres que possuíam ensino médio ou ensino superior completo. Outro fato analisado se referiu à situação conjugal e à área de residência, mas não demonstraram relação com a via de parto realizada. Observou-se que as mães do grupo GC possuem maior escolaridade e renda. Houve maior prevalência de mulheres pertencentes à classe social B2 na realização de cirurgia cesariana e maior prevalência de mulheres da classe social C1 em partos via vaginal. Segundo a literatura, a taxa de cesáreas aumenta com a idade e a renda familiar. Isto pode estar relacionado ao nascimento em hospitais privados, que exibem maiores taxas de cirurgias cesarianas quando comparadas aos hospitais públicos ${ }^{(19)}$.

Dados semelhantes aos da atual pesquisa foram encontrados em um estudo brasileiro, que observou que a cirurgia cesariana esteve mais fortemente associada a melhores condições socioeconômicas, nível de escolaridade e cor de pele (branca) da puérpera, indicando que, quanto mais clara a cor da pele e melhor o nível socioeconômico, maior a proporção de procedimento cirúrgico $^{(19)}$. Em relação à cor de pele, o estudo em questão não observou diferenças, uma vez que, em ambos os grupos, as mães eram predominantemente da raça branca. Em contrapartida, nos Estados Unidos, por exemplo, mulheres brancas e com maior nível socioeconômico realizam mais parto via vaginal ${ }^{(20)}$. Desse modo, quando a mulher pode decidir pela via de parto, a discrepância das taxas de cesárea entre Brasil e Estados Unidos pode revelar a influência de outros fatores, e não somente a cor da pele e o nível socioeconômico, incluindo-se questões biológicas, culturais e sociais influenciando na escolha da via de parto.

Quanto aos fatores de amamentação pesquisados no presente estudo, somente a amamentação na primeira hora de vida demonstrou associação com a via de parto, sendo verificado que quase $80 \%$ das mães do GV amamentaram na primeira hora e que, no GC, apenas $69,53 \%$ conseguiram. Uma das explicações para esse fenômeno pode ser a demora no contato pele a pele da mãe com o bebê e a ocorrência de complicações neonatais antes ou durante a cesariana ${ }^{(7,11)}$, o que também interferiria em amamentar na primeira hora de vida.

Um estudo realizado nos hospitais públicos do Rio de Janeiro, com 673 puérperas, concluiu que o parto vaginal teve um efeito protetor contra o atraso no início da amamentação quando comparado à cesárea ${ }^{(21)}$.

No parto vaginal, o contato direto da mãe com o bebê nos primeiros minutos pós-nascimento contribui no reconhecimento da mãe pelo recém-nascido, o que dá sinais da criança estar pronta para mamar ${ }^{(7,22)}$. A cirurgia cesariana pode ser uma barreira ao início da amamentação, visto que o contato entre mãe e bebê é atrasado devido aos cuidados pósoperatórios ${ }^{(21,23)}$.

A via de parto, por sua vez, no atual estudo, não pareceu ter relação com o tempo de AME e com o sucesso da amamentação. Contudo, sabe-se que o tempo de AME e o sucesso da amamentação sofrem influência de diversos fatores ${ }^{(11)}$, o que pode ter se sobreposto aos dados investigados na pesquisa. Diante disso, salienta-se a importância de estratégias em saúde que visem à promoção do AM e do contato pele a pele entre mãe e bebê na primeira hora após o parto, de modo a favorecer o vínculo mãe/ filho e aumentar as chances de sobrevida neonatal ${ }^{(10)}$.

Este estudo apresentou como limitação o método transversal, pois alguns aspectos abordados poderiam ser obtidos com maior veracidade em um acompanhamento prospectivo. Também pode ter ocorrido viés de memória da participante ao responder ao instrumento de pesquisa.

Recomenda-se a realização de futuros estudos com delineamento longitudinal acerca da relação da via de parto com os fatores investigados, que possam contribuir para melhor entendimento do assunto.

\section{CONCLUSÃO}

Os achados desta pesquisa permitem inferir que a amamentação na primeira hora de vida possui relação com a via de parto ocorrida no grupo estudado. Além disso, as mulheres que realizaram cirurgia cesariana eram prevalentemente casadas, com nível de escolaridade alto e classe social mais alta quando comparadas àquelas que realizaram parto via vaginal.

\section{CONFLITOS DE INTERESSE}

Os autores informam que não houve conflitos de interesse.

\section{CONTRIBUIÇÕES}

Guilherme Tavares de Arruda, Sabrina Cabreira Barreto, Vanessa Lago Morin, Melissa Medeiros Braz, Hedioneia Maria Foletto Pivetta participaram da elaboração da ideia de pesquisa, da coleta de dados e da escrita final do artigo. Gustavo do Nascimento Petter, participou da elaboração da ideia de pesquisa, da análise estatística e da escrita final do artigo.

\section{REFERÊNCIAS}

1. Lagomarsino BS, Van der Sand ICP, Girardon-Perlini NMO, Linck C, Resse LB. A cultura mediando preferências pelo tipo de parto: entrelaçamento de fios pessoais, familiares e sociais. REME. 2013;17(3):680-7. 
2. Ministério da Saúde (BR), Secretaria Executiva. DATASUS: Sistema de Informação de Nascidos Vivos (SINASC) 2018 [acesso em 2018 Abr 5]. Disponível em: http://tabnet.datasus.gov.br/cgi/tabcgi.exe?sinasc/cnv/pnvuf.def

3. Barros FC, Matijasevich A, Maranhão AGK, Escalante JJ, Rabello DL Neto, Fernandes RM, et al. Cesarean sections in Brazil: will they ever stop increasing? Rev Panam Salud Publica. 2015;38(3):217-25.

4. Brasil. Ministério da Saúde. Portaria $n^{\circ} 306$, de 28 de março de 2016. Aprova as Diretrizes de Atenção à Gestante a operação cesariana [acesso em 2017 Set 18]. Disponível em: http://portalsaude.saude.gov.br/images/pdf/2016/marco/31/MINUTAde-Portaria-SAS-Cesariana-03-03-2016.pdf

5. Ministério da Saúde (BR), Comissão Internacional de Incorporação de Tecnologias no SUS. Diretrizes de atenção à gestante: a operação cesariana. Brasília: Ministério da Saúde; 2015 [acesso em 2017 Set 18]. Disponível em: http://conitec.gov.br/ images/Consultas/Relatorios/2015/Relatorio_PCDTCesariana_CP.pdf

6. Sá NNB, Gubert MB, Santos W, Santos LMP. Fatores ligados aos serviços de saúde determinam o aleitamento materno na primeira hora de vida no Distrito Federal, Brasil, 2011. Rev Bras Epidemiol. 2016;19(03):509-24.

7. Esteves TMB, Daumas RP, Oliveira MIC, Andrade CAF, Leite IC. Fatores associados ao início tardio da amamentação em hospitais do Sistema Único de Saúde no Município do Rio de Janeiro, Brasil, 2009. Cad Saúde Pública. 2015;31(11): $2390-400$.

8. Reynolds RD, Pilcher J, Ring A, Johnson R, McKinley P. The Golden Hour: care of the LBW infant during the first hour of life one unit's experience. Neonatal Netw. 2009;28(4):211-19.

9. White-Traut R, Pham T, Yoder J. Exploring factors related to oral feeding progression in premature infants. Adv Neonatal Care. 2013;13(4):288-94.

10. Silva CM, Pereira SCL, Passos IR, Santos LC. Fatores associados ao contato pele a pele entre mãe/filho e amamentação na sala de parto. Rev Nutr. 2016;29(4):457-71.

11. Primo CC, Nunes BO, Lima EFA, Leite FMC, Pontes MB, Brandão MAG. Which factors influence women in the decision to breastfeed? Invest Educ Enferm. 2016;34(1):198-217.

12. Margotti E, Epifanio M. Exclusive maternal breastfeeding and the Breastfeeding Self-efficacy Scale. REME. 2014;15(5): 771-9.

13. Ministério da Saúde (BR), Secretaria de Atenção à Saúde. II Pesquisa de prevalência de aleitamento materno nas capitais brasileiras e Distrito Federal. Brasília: Ministério da Saúde; 2009 [acesso em 2017 Set 19]. Disponível em: http://bvsms. saude.gov.br/bvs/publicacoes/pesquisa_prevalencia_aleitamento_materno.pdf

14. Associação Brasileira de Empresas de Pesquisa. Critério Brasil 2015 e atualização da distribuição de classes para 2016. São Paulo: ABEP; 2014 [acesso em 2017 Set 19]. Disponível em: http://www.abep.org/Servicos/Download.aspx?id=09

15. World Health Organization. Infant and young child feeding: model chapter for textbooks for medical students and allied health professionals. Geneva: WHO Press; 2009 [acesso em 2017 Set 9]. Disponível em: http://goo.gl/DcQWtZ

16. Freitas PF, Fernandes TMB. Associação entre fatores institucionais, perfil da assistência ao parto e as taxas de cesariana em Santa Catarina. Rev Bras Epidemiol. 2016;19(3):525-38.

17. Betrán AP, Ye J, Moller A-B, Zhang J, Gülmezoglu AM, Torloni MR. The increasing trend in caesarean section rates: global, regional and national estimates: 1990-2014. PLoS One. 2016;11(2):e0148343.

18. Rattner D, Moura EC. Nascimentos no Brasil: associação do tipo de parto com variáveis temporais e sociodemográficas. Rev Bras Saúde Mater Infant. 2016;16(1):39-47.

19. Xie RH, Gaudet L, Krewski D, Graham ID, Walker MC, Wen SW. Higher cesarean delivery rates are associated with higher infant mortality rates in industrialized countries. Birth. 2015;42(1):62-9.

20. Gama SGN, Viellas EF, Schilithz AOC, Theme MM Filha, Carvalho ML, Gomes KRO, et al. Fatores associados à cesariana entre primíparas adolescentes no Brasil, 2011-2012. Cad Saúde Pública. 2014;30(Supl 1):117-27.

21. Edmonds JK, Yehezkel R, Liao X, Simas TA. Racial and ethnic differences in primary, unscheduled cesarean deliveries among low-risk primiparous women at an academic medical center: a retrospective cohort study. BMC Pregnancy Childbirth. 2013;13:168.

22. Pereira CRVR, Fonseca VM, Oliveira MIC, Souza IEO, Mello RR. Avaliação de fatores que interferem na amamentação na primeira hora de vida. Rev Bras Epidemiol. 2013;16(2):525-34. 
23. Moore ER, Bergman N, Anderson GC, Medley N. Early skin-to-skin contact for mothers and their healthy newborn infants. Cochrane Database Syst Rev. 2016;11:CD003519.

\section{Endereço para correspondência:}

Guilherme Tavares de Arruda

Universidade Federal de Santa Maria - UFSM

Av. Roraima, 1000 - Cidade Universitária

Bairro: Camobi

CEP: 97105-340 - Santa Maria - RS - Brasil

E-mail: gui_tavares007@hotmail.com 